\title{
SOLVING A BERNOULLI TYPE FREE BOUNDARY PROBLEM WITH RANDOM DIFFUSION
}

\author{
RAHEL BRÜGGER, ROBERTO CROCE, AND HELMUT HARBRECHT
}

\begin{abstract}
The present article is concerned with the numerical solution of a free boundary problem for an elliptic state equation with random diffusion. The domain under consideration is represented by a level set function which is evolved by the objective's shape gradient. The state is computed by the finite element method, where the underlying triangulation is constructed by means of a marching cubes algorithm. The high-dimensional integral, which is induced by the random diffusion, is approximated by the quasi-Monte Carlo method. By numerical experiments, we validate the feasibility of the approach.
\end{abstract}

\section{INTRODUCTION}

Let $T \subset \mathbb{R}^{2}$ denote a bounded domain with free boundary $\partial T=\Gamma$. Inside the domain $T$, we assume the existence of a simply connected subdomain $S \subset T$ with fixed boundary $\partial S=\Sigma$. The resulting annular domain $T \backslash \bar{S}$ is denoted by $D$, see Figure 1 for an illustration. Notice that, instead of a single subdomain $S$, there might also be considered a certain number of simply connected subdomains $S_{i}, i=1, \ldots, k$, which leads to $S=\bigcup_{i=1}^{k} S_{i}$.

Bernoulli's exterior free boundary problem reads as follows. For a given constant $g>0$, seek the domain $D$ and the associated function $u$ such that the overdetermined boundary value problem

$$
\begin{array}{rr}
\Delta u=0 & \text { in } D, \\
u=1 & \text { on } \Sigma, \\
-\frac{\partial u}{\partial \mathbf{n}}=g, u=0 & \text { on } \Gamma,
\end{array}
$$

is satisfied. We like to emphasize that the positivity of the Dirichlet data at the interior boundary implies that $u$ is positive on $D$ and thus it holds in fact $\partial u / \partial \mathbf{n}<0$. Especially, in view of the homogeneous boundary condition at $\Gamma$, we there have the identity $-\partial u / \partial \mathbf{n}=\|\nabla u\|$.

2010 Mathematics Subject Classification. 35R35, 35N25, 65C05, 65N75.

Key words and phrases. Free boundary problem, random diffusion, shape optimization. 


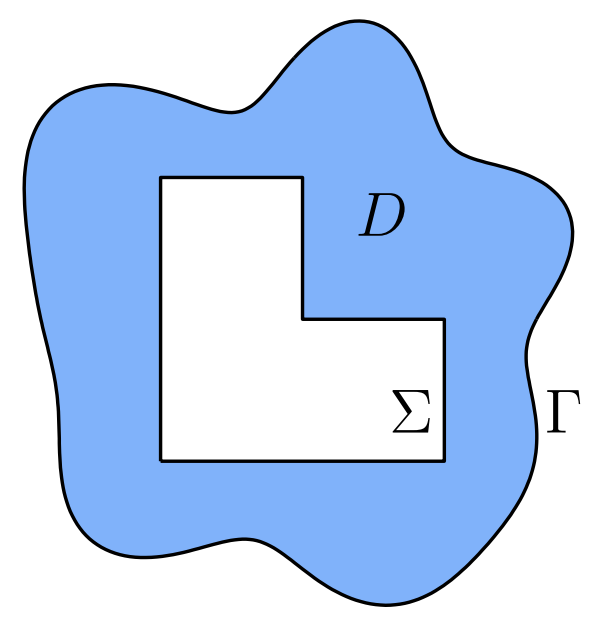

Figure 1 . The domain $D$ and its boundaries $\Gamma$ and $\Sigma$.

The problem under consideration models the growth of anodes in electrochemical processes and can be seen as the prototype of a free boundary problem arising in many applications. For example, in the exterior magnetic shaping of liquid metals, the state equation is the exterior Poisson equation and the uniqueness is ensured by a volume constraint instead by a fixed interior boundary $[9,13,31,36]$.

Shape optimization is a well established tool to solve free boundary problems like (1.1), see $[3,12,14,17,20,22,39,41]$ for example. Here, the free boundary problem is cast into an equivalent minimization problem. For example, the minimizer $D$ of the Dirichlet energy functional

$$
\begin{gathered}
\qquad(D)=\int_{D}\left\{\|\nabla u\|^{2}+g^{2}\right\} \mathrm{d} \mathbf{x} \rightarrow \inf \\
\text { subject to } \Delta u=0 \text { in } D, \quad u=1 \text { on } \Sigma, \quad u=0 \text { on } \Gamma
\end{gathered}
$$

is the sought unknown domain $D$, see [14] for the details. For the existence of solutions to the free boundary problem (1.1), we refer the reader to $[2,4,15]$. Results concerning the geometric form of the solutions can be found in [1] and the references therein.

We will consider a random version of the free boundary problem (1.1) in this article in order to model materials which are not perfectly homogeneous due to imperfections like inclusions of different material or whose material coefficients are uncertain due to measurement errors. To that end, let $(\Omega, \mathcal{F}, \mathbb{P})$ denote an appropriate probability 
space and consider that

$$
\begin{aligned}
& \operatorname{div}(\alpha(\omega) \nabla u(\omega))=0 \quad \text { in } D, \\
& u(\omega)=1 \quad \text { on } \Sigma, \\
& -\alpha(\omega) \frac{\partial u}{\partial \mathbf{n}}(\omega)=g, u(\omega)=0 \quad \text { on } \Gamma,
\end{aligned}
$$

holds for almost all $\omega \in \Omega$. Here, we have in mind a uniformly elliptic random perturbation of the Laplace operator. For solving this random free boundary problem, we will first extend the shape optimization problem (1.2) to elliptic diffusion problems. Especially, we derive the Hadamard representation of the associated shape gradient and prove that the necessary optimality condition imposes a variational formulation of the desired Neumann boundary condition. Hence, we are able to solve (1.3) for each realization $\omega \in \Omega$. Therefore, since the random diffusion induces a random state and thus a random shape functional, we shall minimize the ensemble average of the random shape functional. Notice that we do not consider here other sources of uncertainty like random boundary data or a random interior boundary. These sources of randomness for Bernoulli's free boundary problem have already been considered in $[10,11]$.

The rest of this article is organized as follows. Section 2 is dedicated to the reformulation of the free boundary problem (1.3) as a random shape optimization problem. Then, in Section 3, we introduce the level set method which we will use to represent the sought optimal domain. In Section 4, we present the numerical method to compute the state function. We consider the mesh generation algorithm and briefly recall the computation of the shape gradient by means of the finite element method. In Section 5, we present numerical results to demonstrate the capability of our approach. Especially, it turns out that the random diffusion has indeed a great impact on the solution of the free boundary problem. In the last section, we state concluding remarks.

\section{Shape Optimization For FREe Boundary PROBlems}

2.1. A free boundary problem with non-constant coeffcients. In this section, we will provide the mathematical theory concerning the solution of the free boundary problem under consideration in case of a diffusion coefficient which is non-constant 
but deterministic. Therefore, consider the problem

$$
\begin{array}{rr}
\operatorname{div}(\alpha \nabla u)=0 & \text { in } D, \\
u=1 & \text { on } \Sigma, \\
-\alpha \frac{\partial u}{\partial \mathbf{n}}=g, u=0 & \text { on } \Gamma .
\end{array}
$$

Here, the fixed boundary $\Sigma$ is Lipschitz-continuous and $g>0$ is a given constant. The free boundary $\Gamma$ is sought such that the overdetermined boundary value problem (2.1) admits a solution.

We will denote the hold all by $\mathbb{D} \subset \mathbb{R}^{2}$ and assign $\Upsilon$ to be the set of all annular shapes $D \subset \mathbb{D}$ which have a $C^{2}$-smooth exterior boundary $\Gamma$. Especially, we assume that the diffusion coefficient is sufficiently regular and uniformly positive on the hold all. This means $\alpha \in C^{1}(\mathbb{D})$ and that there exist constants $\underline{\alpha}, \bar{\alpha} \in \mathbb{R}$ such that

$$
0<\underline{\alpha} \leq \alpha \leq \bar{\alpha}<\infty \quad \text { on } \mathbb{D} .
$$

In order to solve the free boundary problem (2.1), we aim at minimize the energy functional

$$
J(D)=\int_{D}\left\{\alpha\|\nabla u\|^{2}+\frac{g^{2}}{\alpha}\right\} \mathrm{d} \mathbf{x} \rightarrow \inf _{D \in \Upsilon}
$$

over the class $\Upsilon$ of admissible shapes, where the state function $u$ solves the boundary value problem

$$
\begin{aligned}
\operatorname{div}(\alpha \nabla u)=0 & \text { in } D, \\
u=1 & \text { on } \Sigma, \\
u=0 & \text { on } \Gamma .
\end{aligned}
$$

Notice that the existence of minimizers for the shape optimization problem (2.3) and (2.4) follows from [7, Theorem 4.2], since we have an energy type shape functional.

2.2. Shape calculus. We briefly recall well known facts about shape calculus, useful for the discussion of the necessary condition and the numerical algorithms. For a general overview on shape calculus, mainly based on the perturbation of identity (Murat and Simon) or the speed method (Sokolowski and Zolésio), we refer the reader to $[12,29,37,38,39]$ and the references therein.

For a smooth perturbation field $\mathbf{U}: \Gamma \rightarrow \mathbb{R}^{2}$, we define the perturbed shape $D_{\varepsilon}$ via its exterior boundary $\Gamma_{\varepsilon}$ in accordance with

$$
\Gamma_{\varepsilon}=\{\mathbf{x}+\varepsilon \mathbf{U}(\mathbf{x}): \mathbf{x} \in \Gamma\}
$$


with $\varepsilon>0$ sufficiently small [29]. This enables the definition of the shape derivative of the shape functional $J$ at $D$ in direction of a vector field $\mathbf{U}$ by

$$
\delta J(D)[\mathbf{U}]=\lim _{\varepsilon \rightarrow 0} \frac{J\left(D_{\varepsilon}\right)-J(D)}{\varepsilon} .
$$

The shape functional $J$ is shape differentiable at $D$, if the Eulerian derivative $\delta J(D)[\mathbf{U}]$ exists for all directions $\mathbf{U}$ and if the mapping $\mathbf{U} \mapsto \delta J(D)[\mathbf{U}]$ is linear and continuous. In particular, according to the Hadamard-Zolésio structure theorem $[12,39]$, it is known that the shape gradient $\nu$ can be expressed as a boundary integral of the form

$$
\delta J(D)[\mathbf{U}]=\int_{\Gamma}\langle\mathbf{U}, \mathbf{n}\rangle \nu \mathrm{d} \sigma .
$$

Furthermore, we shall introduce the local shape derivative $\delta u=\delta u[\mathbf{U}]$ that describes the sensitivity of the state with respect to shape variations. It is defined pointwise by

$$
\delta u(\mathbf{x}):=\lim _{\varepsilon \rightarrow 0} \frac{u_{\varepsilon}(\mathbf{x})-u(\mathbf{x})}{\varepsilon}, \quad \mathbf{x} \in D \cap D_{\varepsilon},
$$

with the solution of the boundary value problem on the perturbed shape denoted by $u_{\varepsilon}$.

Theorem 2.1. Let $\mathbf{n}$ denote the outward unit normal vector to the boundary $\Gamma$ and consider a $C^{2}$-smooth boundary perturbation field $\mathbf{U}: \Gamma \rightarrow \mathbb{R}^{2}$. Then, the Hadamard representation of the shape gradient to the functional (2.3) reads

$$
\delta J(D)[\mathbf{U}]=\int_{\Gamma}\langle\mathbf{U}, \mathbf{n}\rangle\left\{\frac{g^{2}}{\alpha}-\alpha\|\nabla u\|^{2}\right\} \mathrm{d} \sigma .
$$

Proof. Following [39], we obtain

$$
\delta J(D)[\mathbf{U}]=2 \int_{D} \alpha\langle\nabla u, \nabla \delta u\rangle \mathrm{d} \mathbf{x}+\int_{\Gamma}\langle\mathbf{U}, \mathbf{n}\rangle\left\{\alpha\|\nabla u\|^{2}+\frac{g^{2}}{\alpha}\right\} \mathrm{d} \sigma .
$$

Here, the local shape derivative $\delta u=\delta u[\mathbf{U}]$ satisfies

$$
\begin{aligned}
\operatorname{div}(\alpha \nabla \delta u) & =0 & & \text { in } D, \\
\delta u & =0 & & \text { on } \Sigma, \\
\delta u & =-\langle\mathbf{U}, \mathbf{n}\rangle \frac{\partial u}{\partial \mathbf{n}} & & \text { on } \Gamma .
\end{aligned}
$$

By applying integration by parts, one obtains

$$
\begin{aligned}
\int_{D} \alpha\langle\nabla u, \nabla \delta u\rangle \mathrm{d} \mathbf{x} & =-\int_{D} \operatorname{div}(\alpha \nabla u) \delta u \mathrm{~d} \mathbf{x}+\int_{\partial D} \alpha\langle\nabla u, \mathbf{n}\rangle \delta u \mathrm{~d} \sigma \\
& =\int_{\Gamma} \alpha \frac{\partial u}{\partial \mathbf{n}} \delta u \mathrm{~d} \sigma
\end{aligned}
$$


where we inserted in the last step local shape derivative's homogeneous Dirichlet boundary condition at $\Sigma$. Therefore, in view of the local shape derivative's Dirichlet boundary condition at $\Gamma$, we conclude

$$
\int_{D} \alpha\langle\nabla u, \nabla \delta u\rangle \mathrm{d} \mathbf{x}=-\int_{\Gamma} \alpha\langle\mathbf{U}, \mathbf{n}\rangle\|\nabla u\|^{2} \mathrm{~d} \sigma .
$$

Inserting this identity into (2.6) yields the desired shape gradient (2.5).

2.3. Necessary optimality condition. By having the shape gradient at hand, we are able to formulate the following result about the necessary optimality condition of the shape problem under consideration. It shows that the desired Neumann boundary condition is variationally imposed through the shape functional (2.3).

Theorem 2.2. The first order necessary optimality condition for the optimum free boundary $\Gamma^{\star}$ of the minimization problem (2.1) under consideration reads

$$
-\alpha \frac{\partial u}{\partial \mathbf{n}}=g \quad \text { on } \Gamma^{\star} \text {. }
$$

Proof. If $D^{\star} \in \Upsilon$ is an optimizer, then the shape gradient (2.5) vanishes for all sufficiently smooth perturbation fields $\mathbf{U}: \Gamma^{\star} \rightarrow \mathbb{R}$ :

$$
\delta J\left(D^{\star}\right)[\mathbf{U}]=0 \quad \text { for all } \mathbf{U} \in C^{2}\left(\mathbb{R}^{2}\right) .
$$

The fundamental lemma of calculus of variations implies thus

$$
\nu=\frac{g^{2}}{\alpha}-\alpha\|\nabla u\|^{2}=0 \quad \text { on } \Gamma^{\star} .
$$

This is equivalent to the desired necessary condition, due to the uniform positivity (2.2) of $\alpha$ on $D^{\star}$ and due to the maximum principle, implying $u>0$ on $D^{\star}$ and hence $\partial u / \partial \mathbf{n}=-\|\nabla u\|<0$ on $\Gamma^{\star}$.

2.4. Random diffusion. We shall now incorporate random diffusion to the free boundary problem. To this end, let $(\Omega, \mathcal{F}, \mathbb{P})$ be a complete, separable probability space with $\sigma$-field $\mathcal{F} \subset 2^{\Omega}$ and probability measure $\mathbb{P}$. Assume further that the random field $\alpha$ is member of the Bochner space $L_{\mathbb{P}}^{\infty}\left(\Omega ; C^{1}(\mathbb{D})\right)$, satisfying $\mathbb{E}[\alpha] \equiv 1$ and

$$
0<\underline{\alpha} \leq \alpha(\omega) \leq \bar{\alpha}<\infty \quad \text { on } \mathbb{D} .
$$

Then, in accordance with [19], the solution of the random diffusion problem

$$
\begin{aligned}
\operatorname{div}(\alpha(\omega) \nabla u(\omega)) & =0 & & \text { in } D, \\
u(\omega) & =1 & & \text { on } \Sigma, \\
u(\omega) & =0 & & \text { on } \Gamma .
\end{aligned}
$$


satisfies $u \in L_{\mathbb{P}}^{\infty}\left(\Omega ; C^{2}(D)\right)$ for any annular domain $D \in \Upsilon$. Hence, the random shape functional

$$
J(D, \omega)=\int_{D}\left\{\alpha(\omega)\|\nabla u(\omega)\|^{2}+\frac{g^{2}}{\alpha(\omega)}\right\} \mathrm{d} \mathbf{x}
$$

is well-defined, satisfying $J \in L_{\mathbb{P}}^{\infty}(\Omega ; \mathbb{R})$.

According to the previous subsections, the minimization of the shape functional (2.9) subject to (2.8) would yield the solution to the free boundary problem (1.3) for each instance $\omega \in \Omega$. Therefore, in order to define a meaningful solution to the random problem under consideration, we should take the ensemble average of (2.9) and minimize it over the class $\Upsilon$ of admissible shapes:

$$
\mathbb{E}[J(D, \omega)] \rightarrow \inf _{D \in \Upsilon} .
$$

Instead of solving (2.10), one can also minimize the shape functional (2.9) for each particular realization $\alpha(\omega)$ of the diffusion coefficient. This would result in a random domain $D(\omega)$. Besides being computationally extremely expensive, the expectation $\mathbb{E}[D(\omega)]$ of a random domain $D(\omega)$ is no canonic expression but needs to be defined, see [11] for example.

Due to Fubini's theorem, we can interchange the order of integration in (2.10), arriving at

$$
\mathbb{E}[J(D, \omega)]=\int_{D} \int_{\Omega}\left\{\alpha(\omega)\|\nabla u(\omega)\|^{2}+\frac{g^{2}}{\alpha(\omega)}\right\} \mathrm{d} \mathbb{P}(\omega) \mathrm{d} \mathbf{x} .
$$

Our assumption on the random coefficient ensures $u \in L_{\mathbb{P}}^{2}\left(\Omega ; C^{2}(D)\right)$ and, hence, (2.11) is well defined. In particular, it is shape differentiable. In view of Theorem 2.2 , we conclude the following result.

Corollary 2.3. The shape gradient of (2.11) is given by

$$
\delta \mathbb{E}[J(D, \omega)][\mathbf{U}]=\int_{\Gamma}\langle\mathbf{U}, \mathbf{n}\rangle \int_{\Omega}\left\{\frac{g^{2}}{\alpha(\omega)}-\alpha(\omega)\|\nabla u(\omega)\|^{2}\right\} \mathrm{d} \mathbb{P}(\omega) \mathrm{d} \sigma .
$$

The existence of minimizers to the shape optimization problem (2.10) follows again from [7, Theorem 4.2], since the shape functional is of energy type.

\section{Shape Representation Via LeVEL SET FUnCtions}

3.1. Level set functions. The level set method allows to compute the motion of a boundary under a velocity field $\mathbf{V}$, especially for multiply-connected domains, 
cf. [8, 32, 33]. The level set function $\phi: \square \rightarrow \mathbb{R}$, where $\square \subset \mathbb{R}^{2}$ denotes a sufficiently large square, defines the shape $T$ and thus the free boundary $\Gamma=\partial T$ by the rule

$$
\begin{aligned}
& \phi(\mathbf{x})>0 \Rightarrow \mathbf{x} \in T \\
& \phi(\mathbf{x})=0 \Rightarrow \mathbf{x} \in \Gamma \\
& \phi(\mathbf{x})<0 \Rightarrow \mathbf{x} \in \mathbb{R}^{2} \backslash \bar{T} .
\end{aligned}
$$

An important representative of a level set function is the signed distance function, which we will consider in our particular implementation. It has the property

$$
|\phi(\mathbf{x})|=\min _{\mathbf{y} \in \Gamma}\{\|\mathbf{x}-\mathbf{y}\|\}
$$

where its sign tells whether the point $\mathbf{x} \in \mathbb{R}^{2}$ lies inside or outside the shape $T$ in the same convention as in (3.1). Hence, the signed distance function has the same sign property as level set functions, but also fulfills the Eikonal equation

$$
\|\nabla \phi(\mathbf{x})\|=1
$$

almost everywhere. The signed distance function has the advantage that it returns the distance of a point to the boundary, whereas a general level set function only tells us whether a point is inside or outside the shape.

3.2. Level set equation. For solving the shape optimization problem (2.3) and (2.4), we need to add dynamics to the level set function. To this end, we consider the advection-convection equation

$$
\frac{\partial \phi}{\partial t}+\langle\mathbf{V}, \nabla \phi\rangle=0
$$

where $t>0$ is the variable in which the level set function evolves [34]. We shall define a velocity field $\mathbf{V}$ in normal direction, meaning that $\mathbf{V}=\nu \mathbf{n}$ with a scalar function $\nu: \square \rightarrow \mathbb{R}$. Since the relation

$$
\mathbf{n}=\frac{\nabla \phi}{\|\nabla \phi\|} \quad \text { on }\{\phi=0\}
$$

holds for the normal $\mathbf{n}$, we arrive at the level set equation for motion in normal direction:

$$
\frac{\partial \phi}{\partial t}+\nu\|\nabla \phi\|=0 .
$$


3.3. The choice of the velocity field. It is crucial to choose the velocity field $\nu \mathbf{n}$ in the level set equation (3.2) in an appropriate way. The goal is to obtain some decrease in the shape functional under consideration and hopefully convergence to a solution of the optimization problem [8]. We first define $\nu$ on the boundary of the domain by means of the shape gradient defined in (2.12):

$$
\nu=\int_{\Omega}\left\{\frac{g^{2}}{\alpha(\omega)}-\alpha(\omega)\|\nabla u(\omega)\|^{2}\right\} \mathrm{d} \mathbb{P}(\omega) \text { on } \Gamma .
$$

We then need to extend $\nu$ into $\square$ in order to have a contribution of it on the discrete mesh in $\square$ used for numerical computations, cf. [8]. This is done by multiplying $\left.\nu\right|_{\Gamma}$ with an approximation to the delta distribution

$$
\delta(\phi)= \begin{cases}\frac{1}{2}+\frac{1}{2} \cos \left(\frac{\pi \phi}{\varepsilon}\right), & -\varepsilon \leq \phi \leq \varepsilon \\ 0, & \text { otherwise }\end{cases}
$$

leading to a smeared out velocity field

$$
\nu \mathbf{n}=\left.\delta(\phi) \nu\right|_{\Gamma} \mathbf{n}
$$

3.4. Discretization of the transport equation. The discretization of the level set function $\phi$ is performed in a finite difference (FD) framework. The free boundary moves towards the optimal shape via the transport equation (3.2) with motion in normal direction using (3.3) as according velocity strength. A second order TVD Runge-Kutta method is employed for the time discretization and a second order ENO scheme for the space discretization, compare [6, 32, 33]. To this end, we coupled our finite element (FE) method (see the next section) with the Level Set Method Toolbox, cf. [26, 27, 28].

Since the transport equation is explicitly discretized, we have to control the time step $\Delta t$ such that the information is not tracked further than one grid-cell of the FD mesh of size $\Delta x$. Therefore, we use an adaptive time-step control in accordance with

$$
\Delta t=\frac{1}{2 \sqrt{2}} \frac{\Delta x}{\|\nu\|_{\infty}}
$$

to ensure numerical stability for the transport on the FD mesh.

3.5. Reinitialization. In our implementation, we use the reinitialization from [40] to maintain the signed distance property in each step time. If $\phi^{\star}$ is a distorted signed distance function due to the transport of the level set function, then the 
signed distance function with the same zero level set is obtained as the steady state $\phi$ of the Hamilton-Jacobi equation in pseudo time $t$

$$
\frac{\partial \phi}{\partial t}+\operatorname{sign}\left(\phi^{\star}\right)(\|\nabla \phi\|-1)=0
$$

when using the initial value $\phi(\mathbf{x}, 0)=\phi^{\star}(\mathbf{x})$. Equation (3.5) is also discretized by a second order TVD Runge-Kutta method in time and a second order ENO scheme in space. In particular, the sign step-function is approximated in accordance with [35] by

$$
\operatorname{sign}\left(\phi^{\star}\right) \approx \frac{\phi^{\star}}{\sqrt{\left(\phi^{\star}\right)^{2}+\left\|\nabla \phi^{\star}\right\|^{2}(\Delta x)^{2}}} .
$$

Since our numerical approach relies on the signed distance property in the vicinity of the free surface only, it is sufficient to compute the solution of (3.5) in the $\varepsilon$ neighbourhood (3.4) of the zero level set. The parameter $\varepsilon$ must be proportional to the spatial resolution $\Delta x$ of the FD mesh and is $\varepsilon=6 \Delta x$ in our simulations. Since the normal velocities have an absolute value $\leq 1$, the choice $\Delta t=\Delta x / 2$ is appropriate to provide the stability of the explicit time discretization.

\section{Numerical method to COMpute the State}

4.1. Reformulation as parametric problem. The computation of the shape gradient (2.12), which defines the motion of the level set function in accordance with (3.3), amounts to the evaluation of an integral over the sample space $\Omega$. This integral has to be recast first into a deterministic high-dimensional one by parametrizing the random diffusion coefficient $\alpha(\omega)$. To this end, we assume that the random diffusion coefficient is represented by

$$
\alpha(\mathbf{x}, \omega)=1+\sum_{k=1}^{M} \alpha_{k}(\mathbf{x}) Y_{k}(\omega),
$$

where the coefficient functions $\left\{\alpha_{k}(\mathbf{x})\right\}_{k}$ are elements of $C^{1}(D)$ and the random variables $\left\{Y_{k}(\omega)\right\}_{k}$ are independently and uniformly distributed in $[-1 / 2,1 / 2]$. For example, the Karhunen-Loève expansion is of the form (4.1), cf. [24].

The assumption that the random variables $\left\{Y_{k}(\omega)\right\}_{k}$ are stochastically independent implies that the pushforward measure $\mathbb{P}_{\mathbf{Y}}:=\mathbb{P} \mathbf{Y}^{-1}$ with respect to the measurable mapping

$$
\mathbf{Y}: \Omega \rightarrow \square:=[-1 / 2,1 / 2]^{M}, \quad \omega \mapsto \mathbf{Y}(\omega):=\left(Y_{1}(\omega), \ldots, Y_{M}(\omega)\right)
$$

is given by the joint density function 1. With this representation at hand, we can reformulate (3.3) as a deterministic expression where, for ease of notation, we take 
the same function names as before. To that end, we substitute the random variables $Y_{k}$ by the coordinates $y_{k} \in[-1 / 2,1 / 2]$ and intend to compute

$$
\nu=\int_{\square}\left\{\frac{g^{2}}{\alpha(\mathbf{y})}-\alpha(\mathbf{y})\|\nabla u(\mathbf{y})\|^{2}\right\} \mathrm{d} \mathbf{y} \quad \text { on } \Gamma,
$$

where $\operatorname{div}(\alpha(\mathbf{y}) \nabla u(\mathbf{y}))=0$ in $D, \quad u(\mathbf{y})=1$ in $\Sigma, \quad u(\mathbf{y})=0$ on $\Gamma$.

Notice that the integrand in (4.2) depends analytically on the high-dimensional parameter $\mathbf{y}$, see [42] for example. Hence, if the spatial functions $\left\{\alpha_{k}\right\}$ in the expansion (4.1) decay fast enough in the sense of the series $\left\{\left\|\alpha_{k}\right\|_{L^{\infty}(\Omega)}\right\}_{k}$, truncation rank robust quadrature rules are available. In our implementation, we apply the quasiMonte Carlo method, using the Halton sequence, to evaluate the high-dimensional integral in (4.2), see [30, 43] for example. Therefore, we just need to be able to compute the state $u$ for specific realizations $\mathbf{y}_{i} \in \square$ and domains $D \in \mathbb{D}$.

4.2. Triangulation. For solving the state equation and for computing the shape gradient, which enters the definition of the velocity field via (4.2), we shall apply the finite element method. To that end, we employ a mesh generator which is based on the idea of marching cubes as firstly proposed by Lorensen and Cline in [25].

We create a regular grid of quadratic cells of step size $h$. By using the sign of the level set method, each vertex is classified as being inside or outside of the domain. For each edge of the cells which has one endpoint inside the domain and one endpoint outside of the shape, the point on the shape's boundary is determined by applying a bisection algorithm. Connecting all the boundary points yields a polygonal approximation $\Gamma_{h}$ of the boundary $\Gamma$, i.e., the boundary of the zero level set. The interior boundary $\Sigma$ is assumed to be resolved exactly.

A triangulation $\mathcal{T}_{h}=\left\{T_{k}\right\}_{k}$ is finally obtained by dividing all quadrilateral cells into two triangles. Since the triangulation may contain degenerated triangles, it has to be improved in a postprocessing step. This needs only be done in the vicinity of the boundary, as the triangulation is fine in the interior. The particular procedure we use in our implementation has been described in [6].

4.3. Truncated Karhunen-Loève expansion. We shall explain how we compute the Karhunen-Loève expansion of the random diffusion coefficient from a given twopoint covariance function $\operatorname{Cov}\left(\mathbf{x}, \mathbf{x}^{\prime}\right)$. In order to obtain piecewise constant realizations of the random diffusion coefficient on the mesh introduced in Subsection 4.2, we first collocate the two-point covariance function in the midpoints $\left\{\mathbf{x}_{T}\right\}_{T \in \mathcal{T}_{h}}$ of the elements, which leads to a matrix

$$
\mathbf{C}_{h}=\left[\operatorname{Cov}\left(\mathbf{x}_{T}, \mathbf{x}_{T^{\prime}}\right)\right]_{T, T^{\prime} \in \mathcal{T}_{h}} .
$$


The Karhunen-Loève expansion is then derived from computing the eigenpairs $\left(\lambda_{k}, \boldsymbol{\alpha}_{k}\right)$ of the eigenvalue problem

$$
\mathbf{D}_{h} \mathbf{C}_{h} \mathbf{D}_{h} \boldsymbol{\alpha}_{k}=\lambda_{k} \mathbf{D}_{h} \boldsymbol{\alpha}_{k}
$$

where $\mathbf{D}_{h}=[|T|]_{T \in \mathcal{T}_{h}}$ denotes the piecewise constant mass matrix. By using the pivoted Cholesky decomposition, cf. [18], we can easily compute a low-rank approximation of

$$
\mathbf{L}_{h} \mathbf{L}_{h}^{\top} \approx \mathbf{C}_{h}
$$

Since typically the rank $M$ of $\mathbf{L}_{h}$ is much smaller than the number of finite elements, we can simply compute the eigenpairs $\left(\widetilde{\lambda}_{k}, \widetilde{\boldsymbol{\alpha}}\right)$ of the small matrix $\mathbf{L}_{h}^{\top} \mathbf{D}_{h} \mathbf{L}_{h} \in \mathbb{R}^{M \times M}$. As one readily verifies, it holds $\lambda_{k}=\widetilde{\lambda}_{k}$ and $\boldsymbol{\alpha}_{k}=\mathbf{L}_{h} \widetilde{\boldsymbol{\alpha}}_{k}$ for all $k=1, \ldots, M$. We like to emphasize that the overall complexity of computing the Karhunen-Loève expansion is only $\mathcal{O}\left(M^{2} N\right)$ by the algorithm proposed, where $M$ is the rank of the pivoted Cholesky decomposition and $N$ is the number of finite elements, see [18]. In particular, the pivoted Cholesky decomposition can also be applied in case of anisotropic correlation kernels.

4.4. Finite element discretization. Having the triangulation $\mathcal{T}_{h}$ at hand, we can apply the finite element method to solve the state equation in (4.2), necessary for computing the velocity field which drives the level set function. For ease of notation, we assume that the parameter $\mathbf{y}$ is fixed and drop its dependence in the subsequent presentation.

We discretize the variational formulation

seek $u_{0} \in H_{0}^{1}(D)$ such that

$$
\int_{D} \alpha\left\langle\nabla u_{0}, \nabla v\right\rangle \mathrm{d} \mathbf{x}=\int_{D} \alpha\langle\nabla w, \nabla v\rangle \mathrm{d} \mathbf{x} \text { for all } v \in H_{0}^{1}(D)
$$

in the finite element space

$$
V_{h}=\left\{v \in C(D):\left.v\right|_{T} \in \mathcal{P}_{1} \text { for all } T \in \mathcal{T}_{h}\right\}
$$

of globally continuous, piecewise linear ansatz functions on the triangulation $\mathcal{T}_{h}$. Here, $w \in H^{1}(D)$ denotes a suitable extension of the Dirichlet data 1 on $\Sigma$ into the domain $D$.

We denote the nodal basis in $V_{h}$ by $\left\{\varphi_{k}: k \in \Lambda\right\}$, where the index set $\Lambda=\Lambda_{\Gamma} \cup \Lambda_{0} \cup \Lambda_{\Sigma}$ consists of nodes on the boundary $\Gamma$, interior nodes, and nodes on the boundary $\Sigma$. A suitable extension of the Dirichlet data at $\Sigma$ is just given by $w_{h}=\sum_{\ell \in \Lambda_{\Sigma}} \varphi_{\ell} \in V_{h}$. 
By making the ansatz $u_{0, h}=\sum_{k \in \Lambda_{0}} u_{k} \varphi_{k} \in V_{h}$ and setting $\mathbf{u}_{h}=\left[u_{k}\right]_{k \in \Lambda_{0}}$, we arrive at the system of linear equations

$$
\mathbf{A}_{h} \mathbf{u}_{h}=\mathbf{f}_{h}
$$

where

$$
\mathbf{A}_{h}=\left[\int_{D} \alpha\left\langle\nabla \varphi_{\ell}, \nabla \varphi_{k}\right\rangle \mathrm{d} \mathbf{x}\right]_{k, \ell \in \Lambda_{0}}, \quad \mathbf{f}_{h}=\left[\sum_{\ell \in \Lambda_{\Sigma}} \int_{D} \alpha\left\langle\nabla \varphi_{\ell}, \nabla \varphi_{k}\right\rangle \mathrm{d} \mathbf{x}\right]_{k \in \Lambda_{0}} .
$$

This system of linear equations can be solved in nearly linear time when using nested dissection, see e.g. [5, 16, 21, 23] and the references therein. It yields an approximation $u_{h}=u_{0, h}+w_{h}$ to the solution of (2.4) which converges quadratically in $h$ with respect to the mesh size $h$ provided that the domain $D$ is convex.

\section{NumERICAL EXPERIMENTS}

5.1. First example. In our first example, we consider the L-shape

$$
S:=(-1 / 2,1 / 2)^{2} \backslash[0,1 / 2)^{2}
$$

as interior domain $S$. The random diffusion coefficient $\alpha$ has the mean value $\mathbb{E}[\alpha] \equiv 1$ and the two-point covariance function

$$
\operatorname{Cov}[\alpha]\left(\mathbf{x}, \mathbf{x}^{\prime}\right)=\frac{3}{5} \exp \left(-\left\|\mathbf{x}-\mathbf{x}^{\prime}\right\|^{2}\right) .
$$

Given the covariance function (5.1), the expansion (4.1) is approximately computed with the help of the pivoted Cholesky decomposition in accordance with Subsection 4.3 up to an accuracy of $10^{-3}$. The expansion rank $M$ is then about 40 . We further apply 10000 Halton points in the quasi-Monte Carlo method to evaluate the high-dimensional integral in (4.2).

The level set function is discretized on the square $\square:=[-2,2]^{2}$ on a rectangular grid of $160 \times 160$ grid cells. The $\varepsilon$ smoothing region for the Dirac functional (3.4) is set to $\varepsilon=6 \Delta x$ while the number of iterations for the reinitialization (3.5) is set to 12 . The signed distance function which corresponds to the circle of radius 1.0, centered in the origin, is used as initialization for the level set function. Then, we perform 200 time steps of the level set method which is enough to get the stationary solution.

The mesh generation for the finite element method is based on the grid which underlies the level set function. This yields a finite element mesh of mesh size about 1/100, which corresponds to about 10-20 000 finite elements, depending on the actual shape. 

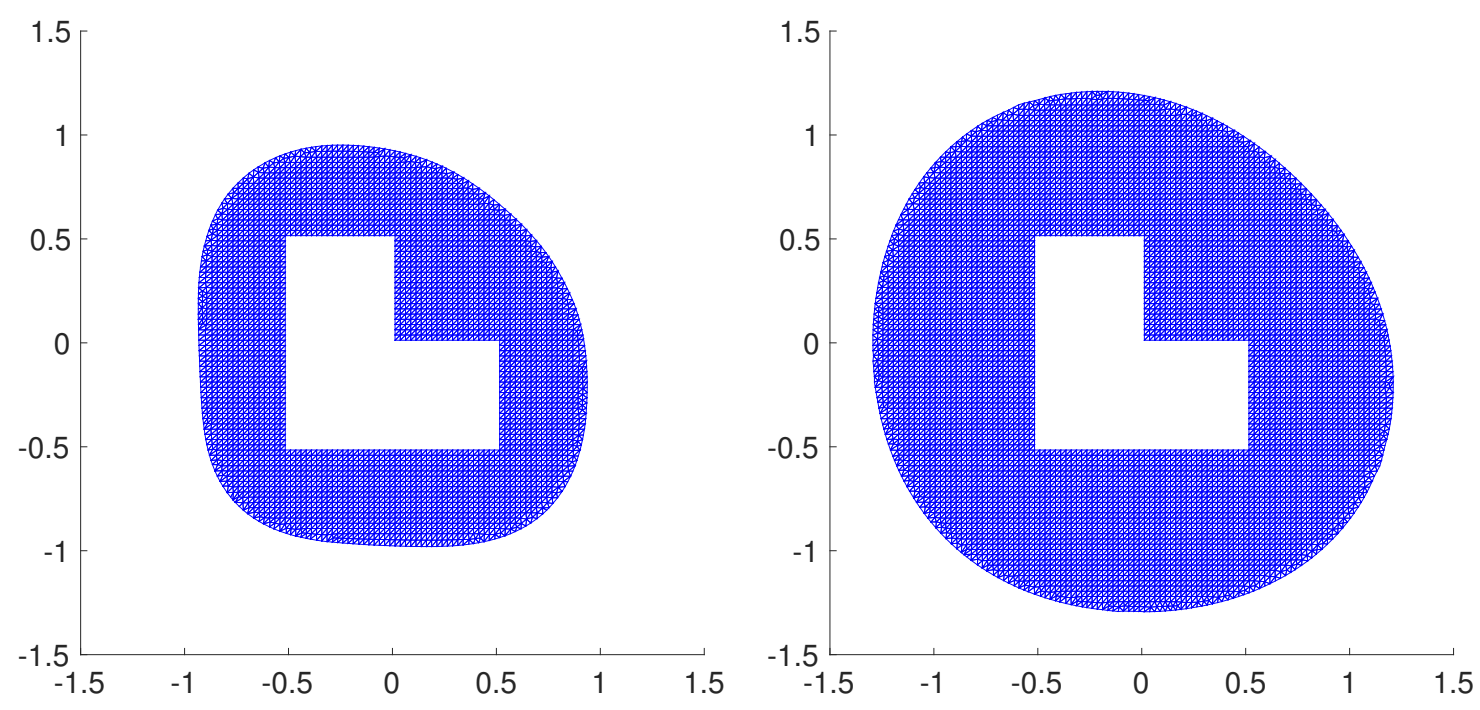

FiguRE 2. Solution to the free boundary problem with random diffusion (left) and without (right) for $g=1$ and an L-shape as fixed inclusion.
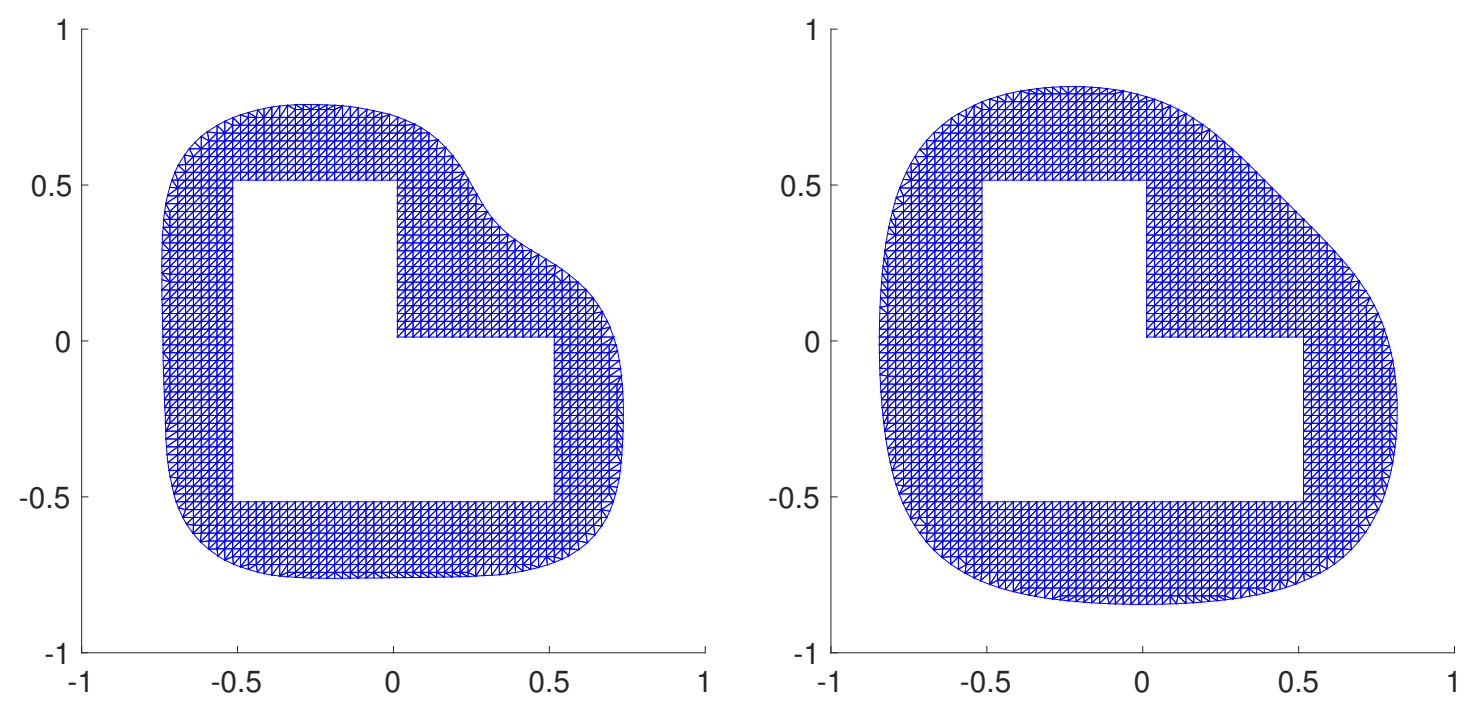

Figure 3 . Solution to the free boundary problem with random diffusion (left) and without (right) for $g=3$ and an L-shape as fixed inclusion.

The prescribed flux $g$ through the free boundary $\Gamma$ is varied in accordance with $g=1, g=3$, and $g=5$, cf. (1.1). The computed free boundaries are found in Figures $2-4$ on the left hand side. In addition to the random diffusion coefficient, we also compute the free boundaries for the deterministic diffusion coefficient $\alpha \equiv 1$. 

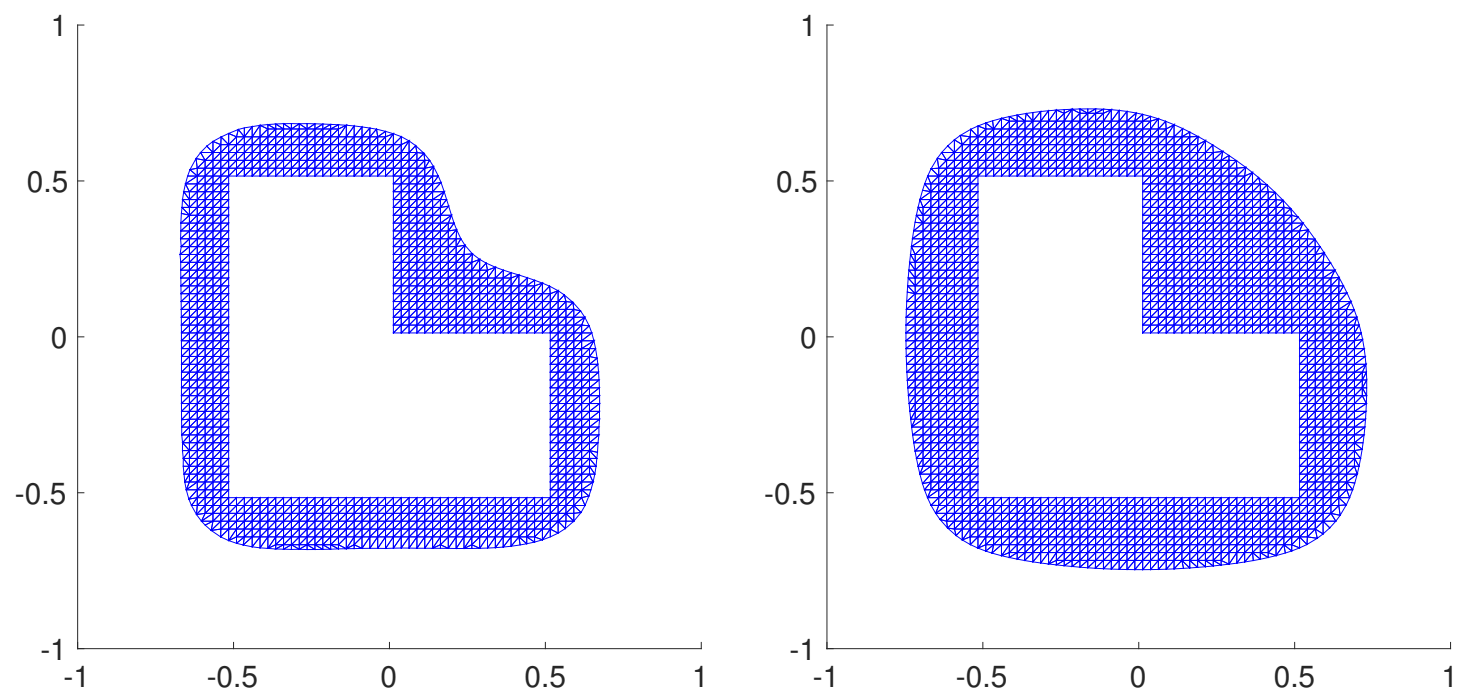

FiguRE 4. Solution to the free boundary problem with random diffusion (left) and without (right) for $g=5$ and an L-shape as fixed inclusion.

The respective results are found in Figures 2-4 on the right hand side. As one can clearly see, the randomness of the diffusion coefficient influences the results drastically, especially in the case $g=1$, cf. Figure 2, where the optimum shape is much smaller than for the deterministic diffusion coefficient. Nonetheless, also for the random diffusion coefficient, we observe that the free boundary approaches the L-shape as the prescribed flux $g$ increases.
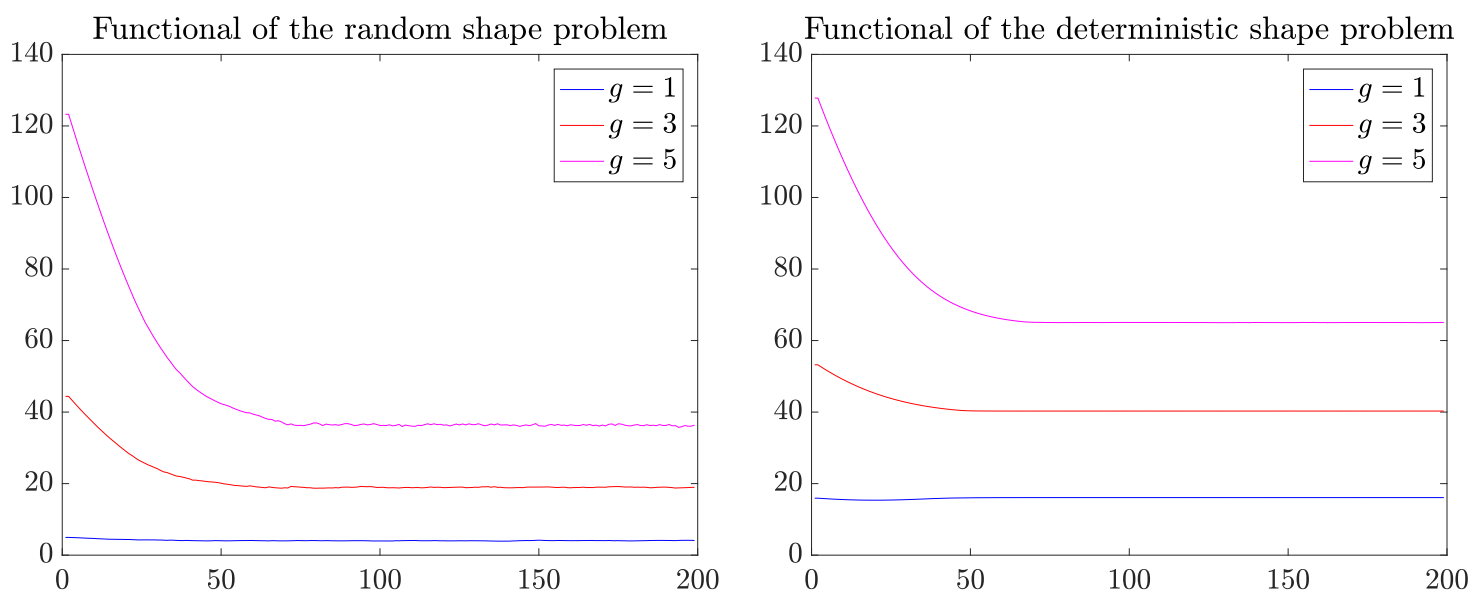

FIGURE 5. Histories of the functional for the random shape optimization problem and for the deterministic shape optimization problem in the case of the L-shape inclusion. 
In Figure 5, we plotted the histories of the respective functionals in case of the deterministic shape optimization problem and the random shape optimization problem. It can be observed by comparing the initial values that the randomness decreases the value of the shape functional. The values of the shape functional are also during the course of minimization smaller in the case of the random diffusion coefficient compared to the deterministic diffusion coefficient, resulting in a smaller minimum value. Nonetheless, the convergence behaviour is rather similar.

5.2. Second example. In the second example, we will treat the situation of two subdomains inside the domain $T$. Thus, if the desired Neumann data are large enough, we expect that the optimum shape consists of two separated components. The level set method is able to deal with such a change of topology.

Consider the two squares

$$
S_{1}=(-1,-1 / 2) \times(-1 / 2,1 / 2), \quad S_{2}=(1 / 2,1) \times(-1 / 2,1 / 2), \quad S=S_{1} \cup S_{2},
$$

to define the two non-connected parts $\partial S_{1}$ and $\partial S_{2}$ of the interior boundary $\Sigma=$ $\partial S_{1} \cup \partial S_{2}$. The random diffusion coefficient $\alpha$ under consideration has again the mean value $\mathbb{E}[\alpha] \equiv 1$ and again the covariance given by (5.1). The expansion rank in (4.1) is about 60 to approximate the two-point covariance up to an accuracy of $10^{-3}$. All the other parameters for the quasi-Monte Carlo method, the level set method, and the finite element method are identical to the settings in the previous example. The only difference is that the initial circle has radius 1.5 and the we need now about 400 time steps of the level set method to get the optimum shape. The additional iterations account for the topological change which appears during the course of the level set method.

We vary again the prescribed Neumann data and compute the resulting free boundary for the random diffusion coefficient and for the deterministic diffusion coefficient $\alpha \equiv 1$. The resulting free boundaries are found in Figure 6 for $g=1$, in Figure 7 for $g=2$, and in Figure 8 for $g=3$, for the random diffusion coefficient on the left in each case and for the deterministic diffusion coefficient on the right in each case.

In case of the deterministic diffusion coefficient, we see that the flux $g=3$ is sufficiently large to split the optimum domain into two components. In case of the random diffusion coefficient, this already happens for $g=2$. In case of the random diffusion coefficient and for $g=2$ and $g=3$, it is clearly observed that the two components are not uncorrelated, compare the left plot in Figure 7 and Figure 8, respectively. This issues from the chosen random model, since the random diffusion 

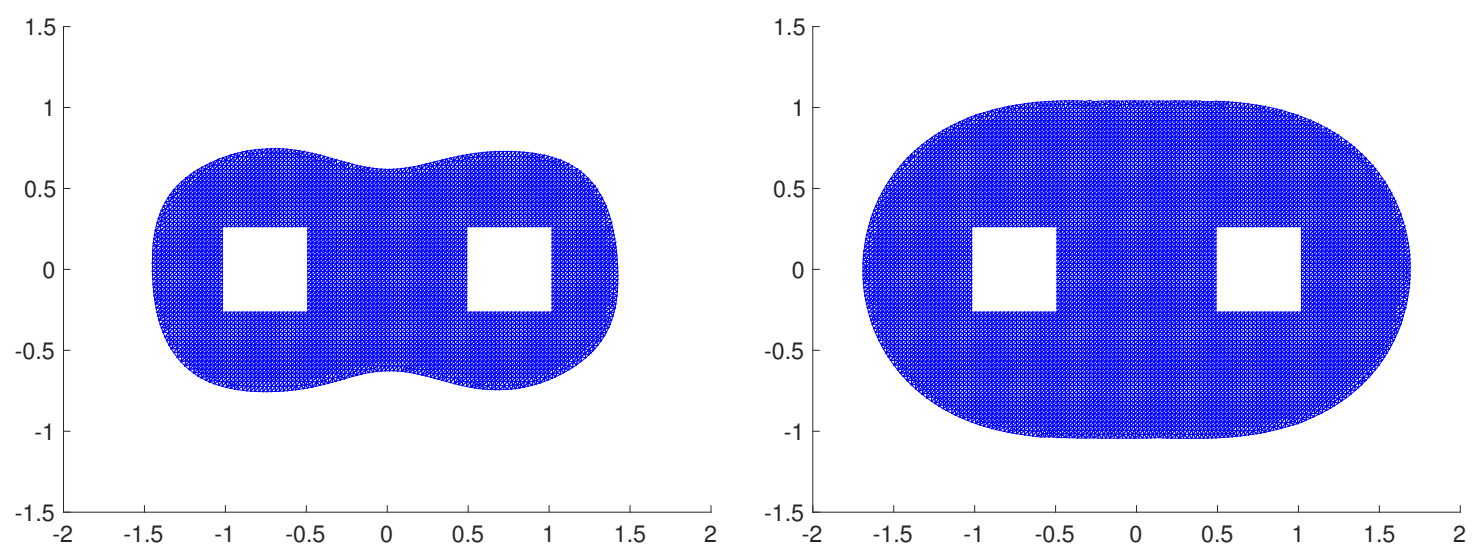

Figure 6. Solution to the free boundary problem with random diffusion (left) and without (right) for $g=1$ and two squares as fixed inclusions.
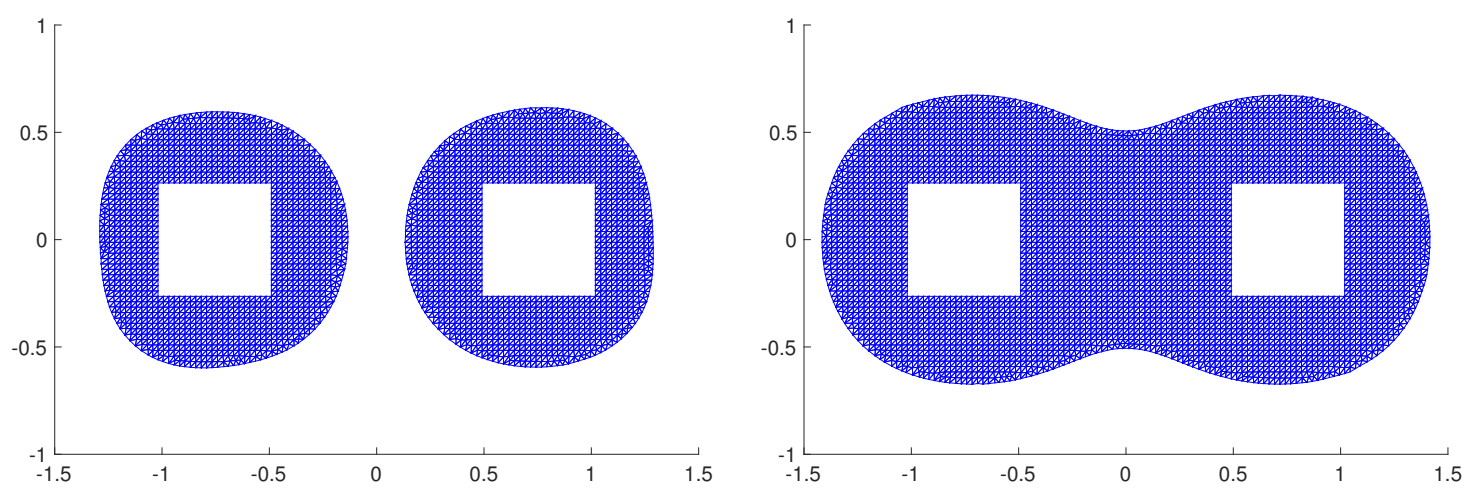

FiguRE 7 . Solution to the free boundary problem with random diffusion (left) and without (right) for $g=2$ and two squares as fixed inclusions.

coefficient in the left and in the right component is correlated according to the choice (5.1).

The histories of the respective functionals in case of the deterministic shape optimization problem and the random shape optimization problem are found in Figure 9. The observations are similar as in the first example. The values of the functional in case of the deterministic shape optimization problem are always larger than in case of the random shape optimization problem while the convergence behaviour is quite similar. 

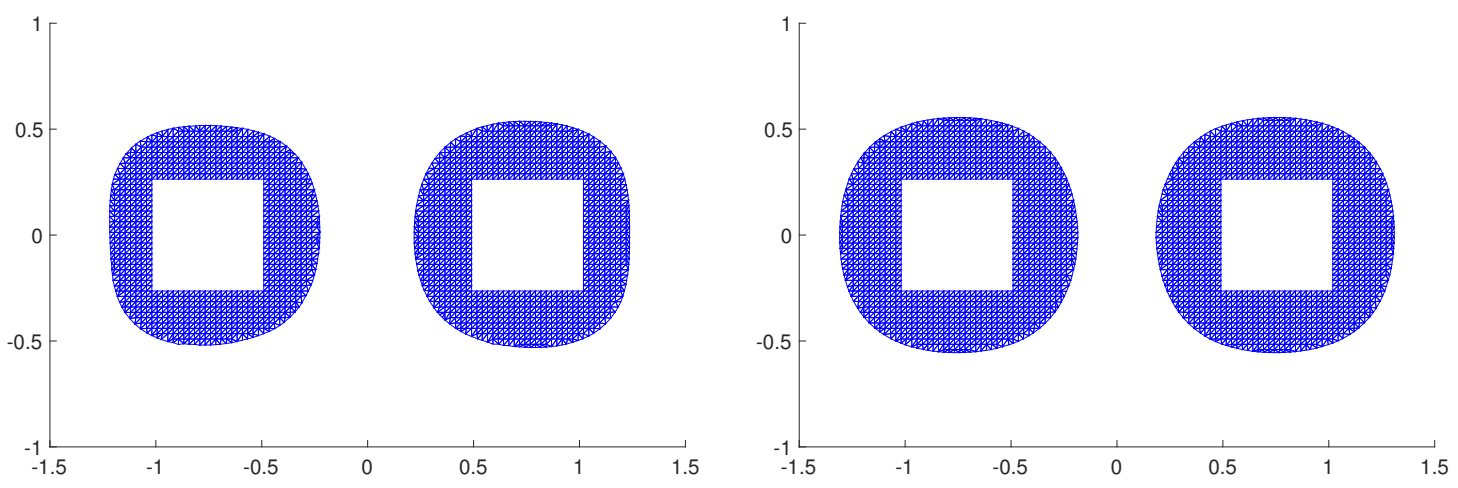

Figure 8. Solution to the free boundary problem with random diffusion (left) and without (right) for $g=3$ and two squares as fixed inclusions.
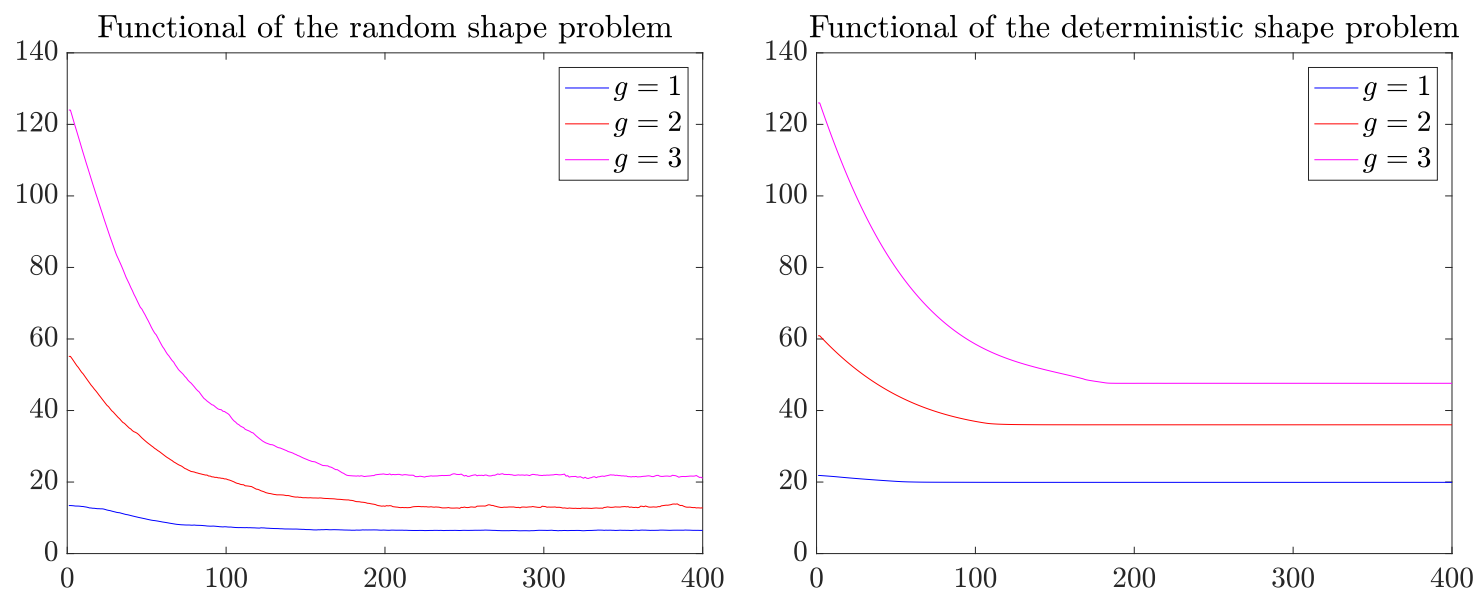

FIGURE 9. Histories of the functional for the random shape optimization problem and for the deterministic shape optimization problem in the case of the two square inclusions.

\section{Conclusion}

In the present article, Bernoulli's free boundary problem has been considered in case of random diffusion. This situation accounts for uncertainties in the material properties under consideration. We have modelled the problem under consideration by minimizing an expected energy functional over the class of admissible shapes. The minimization is performed by a gradient based shape optimization algorithm. After parametrizing the random diffusion coefficient by means of a finite Karhunen-Loève expansion, the shape gradient computation amounts to the evaluation of a highdimensional integral. This integral is evaluated by the quasi-Monte Carlo method. 
The level set method has been used to represented the sought optimum free boundary while the state equation is approximated by the finite element method. Our numerical results show that the randomness has a drastic impact on the optimum free boundary.

\section{REFERENCES}

[1] A. Acker. On the geometric form of Bernoulli configurations. Math. Meth. Appl. Sci., 10:1-14, 1988.

[2] H.W. Alt and L.A. Caffarelli. Existence and regularity for a minimum problem with free boundary. J. Reine Angew. Math., 325:105-144, 1981.

[3] K. Bandara, F. Cirak, G. Of, O. Steinbach, and J. Zapletal. Boundary element based multiresolution shape optimisation in electrostatics J. Comput. Phys., 297:584-598, 2015.

[4] A. Beurling. On free boundary problems for the Laplace equation. Seminars on Analytic functions, Institute for Advanced Study, Princeton, NJ, 1:248-263, 1957.

[5] I. Brainman and S. Toledo. Nested-dissection orderings for sparse LU with partial pivoting. SIAM J. Matrix Anal. Appl., 23:998-1012, 2002.

[6] R. Brügger, R. Croce, and H. Harbrecht. Solving a free boundary problem with nonconstant coefficients. Math. Meth. Appl. Sci., 41(10):3653-3671, 2018.

[7] D. Bucur. How to prove existence in shape optimization. Control Cybernet., 34(1):103$116,2005$.

[8] M. Burger. A framework for the construction of level set methods for shape optimization and reconstruction. Interfaces Free Bound., 5:301-329, 2003.

[9] O. Colaud and A. Henrot. Numerical approximation of a free boundary problem arising in electromagnetic shaping. SIAM J. Numer. Anal., 31: 1109-1127, 1994.

[10] M. Dambrine, C. Dapogny, and H. Harbrecht. Shape optimization for quadratic functionals and states with random right-hand sides. SIAM J. Control Optim., 53(5):3081$3103,2015$.

[11] M. Dambrine, H. Harbrecht, M. Peters, and B. Puig. On Bernoulli's free boundary problem with a random boundary. Int. J. Uncertain. Quantif., 7(4):335-353, 2017.

[12] M. Delfour and J.-P. Zolésio. Shapes and Geometries. SIAM, Philadelphia, 2001.

[13] K. Eppler and H. Harbrecht. Exterior Electromagnetic Shaping using Wavelet BEM. Math. Meth. Appl. Sci., 28:387-405, 2005.

[14] K. Eppler and H. Harbrecht. Efficient treatment of stationary free boundary problems. Appl. Numer. Math., 56:1326-1339, 2006.

[15] M. Flucher and M. Rumpf. Bernoulli's free-boundary problem, qualitative theory and numerical approximation. J. Reine Angew. Math., 486:165-204, 1997.

[16] A. George. Nested dissection of a regular finite element mesh. SIAM J. Numer. Anal., 10:345-363, 1973. 
[17] H. Harbrecht. A Newton method for Bernoulli's free boundary problem in three dimensions. Computing, 82(1):11-30, 2008.

[18] H. Harbrecht, M. Peters, and R. Schneider On the low-rank approximation by the pivoted Cholesky decomposition. Appl. Numer. Math., 62(4):428-440, 2012.

[19] H. Harbrecht, M. Peters, and M. Siebenmorgen. On the quasi-Monte Carlo quadrature with Halton points for elliptic PDEs with log-normal diffusion. Math. Comput., 86:771-797, 2017.

[20] J. Haslinger, T. Kozubek, K. Kunisch and G. Peichl. Shape optimization and fictitious domain approach for solving free boundary value problems of Bernoulli type. Computational Optimization and Applications, 26:231-251, 2003.

[21] B. Hendrickson and E. Rothberg. Improving the run time and quality of nested dissection ordering. SIAM J. Sci. Comput., 20:468-489, 1998.

[22] K. Ito, K. Kunisch, and G. Peichl. Variational approach to shape derivatives for a class of Bernoulli problems. J. Math. Anal. Appl. 314 (2006) 126-149.

[23] R.J. Lipton, D.J. Rose, and R.E. Tarjan. Generalized nested dissection. SIAM J. Numer. Anal., 16:346-358, 1979.

[24] M. Loève. Probability theory. I+II, Graduate Texts in Mathematics 45, Springer, New York, 4th ed., 1977.

[25] W.E. Lorensen and H.E. Cline. Marching cubes: A high resolution 3d surface construction algorithm. SIGGRAPH Comput. Graph., 21(4):163-169, 1987.

[26] I.M. Mitchell. The flexible, extensible and efficient toolbox of level set methods. $J$. Sci. Comput., 35(2-3):300-329, 2008.

[27] I.M. Mitchell. A toolbox of level set methods (version 1.1). Department of Computer Science, University of British Columbia, Vancouver, Canada, Tech. Rep. TR-2007-11, June 2007. http://www.cs.ubc.ca/ ${ }^{\sim m i t c h e l l / T o o l b o x L S / t o o l b o x L S . p d f ~}$

[28] I.M. Mitchell and J.A. Templeton. A toolbox of Hamilton-Jacobi solvers for analysis of nondeterministic continuous and hybrid systems. In Hybrid Systems: Computation and Control, edited by M. Morari and L. Thiele. Lect. Notes Comput. Sci. 3414, Springer, Berlin, pages 480-494, 2005.

[29] F. Murat and J. Simon. Étude de problèmes d'optimal design. In Optimization Techniques, Modeling and Optimization in the Service of Man, edited by J. Céa. Lect. Notes Comput. Sci. 41, Springer, Berlin, pages 54-62, 1976.

[30] H. Niederreiter. Random Number Generation and Quasi-Monte Carlo Methods. Society for Industrial and Applied Mathematics, Philadelphia, PA, 1992.

[31] A. Novruzi and J.-R. Roche. Newton's method in shape optimisation: a threedimensional case. BIT, 40:102-120, 2000.

[32] S. Osher and J.A. Sethian. Fronts propagating with curvature-dependent speed: Algorithms based on Hamilton-Jacobi formulations. J. Comput. Phys., 79(1):12-49, 1988.

[33] S. Osher and R. Fedkiw. Level set methods: An overview and some recent results. J. Comput. Phys., 169(2):463-502, 2001. 
[34] S. Osher and R. Fedkiw. Level Set Methods and Dynamic Implicit Surfaces. Springer, New York, 2003.

[35] D. Peng, B. Merriman, S. Osher, H. Zhao, and M. Kang, A PDE-based fast local level set method. J. Comput. Phys., 155:410-438, 1999.

[36] M. Pierre and J.-R. Roche Numerical simulation of tridimensional electromagnetic shaping of liquid metals. Numer. Math., 65: 203-217, 1993.

[37] O. Pironneau. Optimal Shape Design for Elliptic Systems. Springer, New York, 1983.

[38] J. Simon. Differentiation with respect to the domain in boundary value problems. Numer. Funct. Anal. Optim., 2:649-687, 1980.

[39] J. Sokolowski and J.-P. Zolésio. Introduction to Shape Optimization. Springer, Berlin, 1992.

[40] M. Sussman, P. Smereka, and S. Osher. A level set approach for computing solutions to incompressible two-phase flow. J. Comput. Phys., 114:146-159, 1994.

[41] T. Tiihonen. Shape optimization and trial methods for free-boundary problems. RAIRO Model. Math. Anal. Numér., 31(7):805-825, 1997.

[42] R. Todor and C. Schwab. Convergence rates for sparse chaos approximations of elliptic problems with stochastic coefficients. IMA J. Numer. Anal., 27(2):232-261, 2007.

[43] X. Wang. A constructive approach to strong tractability using quasi-Monte Carlo algorithms. J. Complex., 18:683-701, 2002.

Rahel Brügger, Roberto Croce, Helmut Harbrecht, Departement Mathematik und Informatik, Universität Basel, Spiegelgasse 1, 4051 Basel, Schweiz.

E-mail address: $\{$ ra.bruegger, roberto.croce, helmut.harbrecht\}@unibas.ch 